\title{
A Relevant Inrush Current Limitation Based on SCRs' Smart Control Used in EV Battery Chargers
}

\author{
S. Jacques ${ }^{1}$, C. Reymond ${ }^{1,2}$, G. Benabdelaziz ${ }^{2}$, and J.-C. Le Bunetel ${ }^{1}$ \\ ${ }^{1}$ University of Tours (France), Research group on materials, microelectronics, acoustics, and nanotechnology \\ GREMAN CNRS UMR 7347 \\ 7 avenue Marcel Dassault, 37200 Tours (France) \\ Phone: +33 247 361324, e-mail: sebastien.jacques@univ-tours.fr, lebunetel@univ-tours.fr \\ 2 Application and System Engineering, STMicroelectronics Tours SAS \\ 10 rue Thalès de Milet, 37100 Tours (France) \\ Phone: +33 247 424000, e-mail: cedric.reymond@st.com, ghafour.benabdelaziz@st.com
}

\begin{abstract}
In this article, an active inrush current limiter based on the phase shift control of silicon controlled rectifiers (SCRs) is proposed. These SCRs are used in medium power AC-DC converters (up to $3.7 \mathrm{~kW}$ ) for electric vehicle (EV) charging systems. The control strategy consists in adjusting the triggering delay of SCRs both to limit the peak value of inrush current spikes and accelerate the charge of the DC-link capacitor of the converter. A modeling of the AC-DC converter, based on mathematical calculations, is necessary to implement the control strategy of the SCRs. Experimental test results demonstrate the robustness of the method, and confirm the modeling results.
\end{abstract}

\section{Key words}

AC-DC power supply, medium power EV charger, SCR, phase shift control.

\section{Introduction}

Nowadays, electric vehicles (EVs) are very popular. In particular, EVs contribute to get rid of air pollutants (e.g., $\mathrm{CO}_{2}, \mathrm{SO}_{2}$ and $\mathrm{NO}_{\mathrm{x}}$ ) caused by burning fossil fuels, and bring fuel economy to millions of drivers $[1,2]$.

In recent years, hybrid EVs (HEVs) have been developed to combine the use of an internal combustion engine with one or more electric motors (EMs) connected to a battery pack. Such hybridization improves the fuel economy, but all of the available energy still comes from the fuel tank [3].

Plug-in HEVs (PHEVs) have then been introduced to displace petroleum energy with multi-source electrical energy [4]. So, PHEVs are able to draw power from the electric grid, store it in batteries, and use it for transportation. These batteries play an important role due to their cost-effectiveness, energy and power densities, reliability, and charging time that depend on practical applications [5]. In particular, the lifetime and charging duration strongly depend on the features of the battery charger.

Two kinds of battery chargers are currently used. The first one is the on-board type. The second one is the standalone type that provides fast charging. Regarding the on-board type, many authors have recently pointed out the importance to design a high power and high efficiency battery charger, while optimizing its weight, bulk and cost, because it should be carried by the vehicle [6].

Medium power (e.g., $3.7 \mathrm{~kW}$ ) on-board battery charger converts alternating current (AC) from widely used AC $110 \mathrm{~V}-230 \mathrm{~V} / 16 \mathrm{~A}$ outlet to DC $200 \mathrm{~V}-430 \mathrm{~V} / 10 \mathrm{~A}$ in order to charge the batteries by controlling the voltage and current flow.

This kind of charger is typically composed of a large bulk electrolytic capacitance (e.g., $1 \mu \mathrm{F} / \mathrm{W}$ for $230 \mathrm{~V}$ RMS (root mean square), $50 \mathrm{~Hz} \mathrm{AC}$ mains) [7]. This output capacitor, also called DC-link capacitor, is responsible for smoothing ripple in the rectified current prior being chopped at a high frequency.

When the AC-DC power supply is plugged in, high inrush currents can be generated on AC-mains due to the charge of the output capacitor. Such high currents can easily range from 5 times to 20 times higher than the steady state load current [8].

There are many consequences of these phenomena. Firstly, the equipment itself can be affected (e.g., blown fuses or tripped circuit breakers). Secondly, the individual devices of the AC-DC power supply (such as switches, rectifier diodes, smoothing capacitors) can be damaged. Thirdly, high inrush currents can induce an excessive current stress on AC-mains. 
The research group on materials, microelectronics, acoustics and nanotechnology (GREMAN CNRS UMR 7347, University of Tours, France), together with STMicroelectronics Tours SAS, has recently proposed an active inrush current limitation technique [9]. This technique is based on the phase shift control of silicon controlled rectifiers (SCRs) used in a medium power ACDC converter.

This paper serves several purposes. First of all, the typical topology of AC-DC converter used in an on-board battery charger is recalled. The aim is to point out the relevance of the use of SCRs. Then, the smart control strategy of the thyristors is explained. Finally, experimental measurement results are discussed both to highlight the robustness of this method, and ensure that the AC-DC converter operates accurately.

\section{Example of Typical EV Battery Charger Topology and Active ICL Proposal using SCRs}

Figure 1 shows a typical topology of on-board EV charger. In that case, the AC-DC stage is composed of a totem-pole power factor corrector (PFC) coupled with a resonant LLC converter. In comparison with traditional $\mathrm{PFC}$, for each AC-line half cycle, the totem-pole PFC uses one diode for the power-conduction path instead of 2. As a consequence, the efficiency of the converter is better because the conduction losses can be reduced. The resonant LLC converter is helpful to warrant high efficiency, low electromagnetic interferences (EMI) and high power density. However, its design may require more effort for optimization in comparison with pulse width modulation (PWM) converters [10]. One typical solution to build an inrush current limiter (ICL) consists in associating negative temperature coefficient (NTC) thermistor in parallel with a bypass switch such as an electromechanical relay. This parallel system is in series with AC-line. Such ICL induces several drawbacks. Firstly, the thermistor must be cooled down to reset it to high resistive mode. The recovery time is achieved by the bypass switch. An electromechanical relay has particularly many major drawbacks: bulky solution, high current consumption of the coil, risk of relay opening in case of vibrations, risk of explosion in flammable environment.

In this paper, we propose to replace the two diodes of the totem-pole PFC (see Figure 1) by 2 SCRs to create a soft starter. In that case, the NTC thermistor coupled with the by-pass switch can be removed. Such solution is helpful both to limit inrush currents and optimize the charge of the DC-link capacitance by controlling the phase shift of each SCR. In particular, in this article, some tips are given to optimize the phase shift angle. A control algorithm is used and implemented in a microcontroller unit (MCU) to meet the expectations described above.

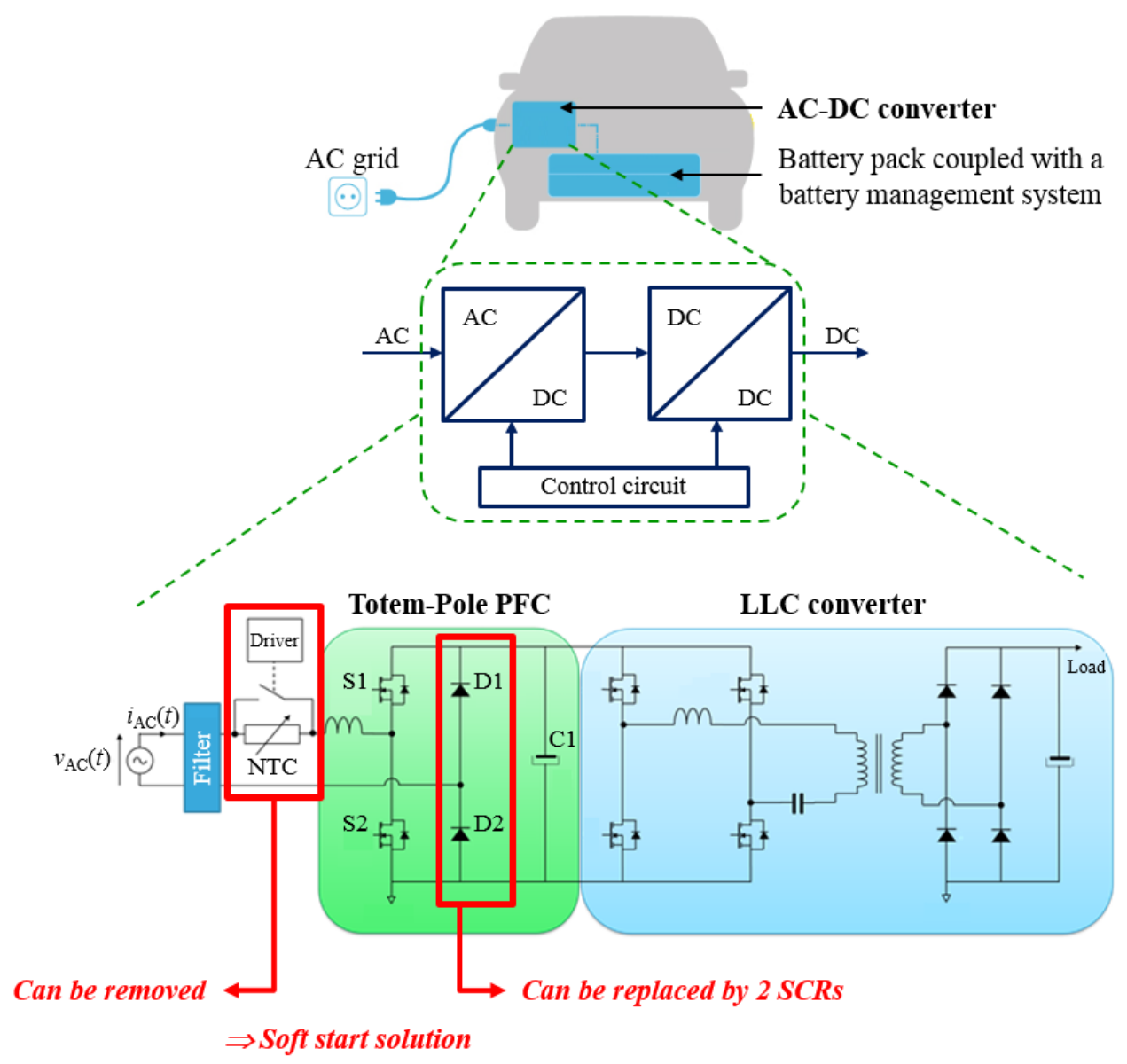

Fig. 1. Typical EV on-board battery charger topology based on a totem-pole PFC. Soft start function proposal using SCRs. 


\section{Soft Start Principle}

As can be seen in Figure 2, the first control strategy of the SCRs is based on a constant triggering delay $(\Delta t)$. The gate current pulse width (PW) is incremented at each SCR turnon. So, it depends on the $\Delta t$-parameter. In particular, the duration of each consecutive pulse is increased with the $\Delta t$-value. As a consequence, it is possible to control the charge of the DC-link capacitor (see Figure 1, the DC-link capacitor is named " $\mathrm{C} 1$ ") using this method.

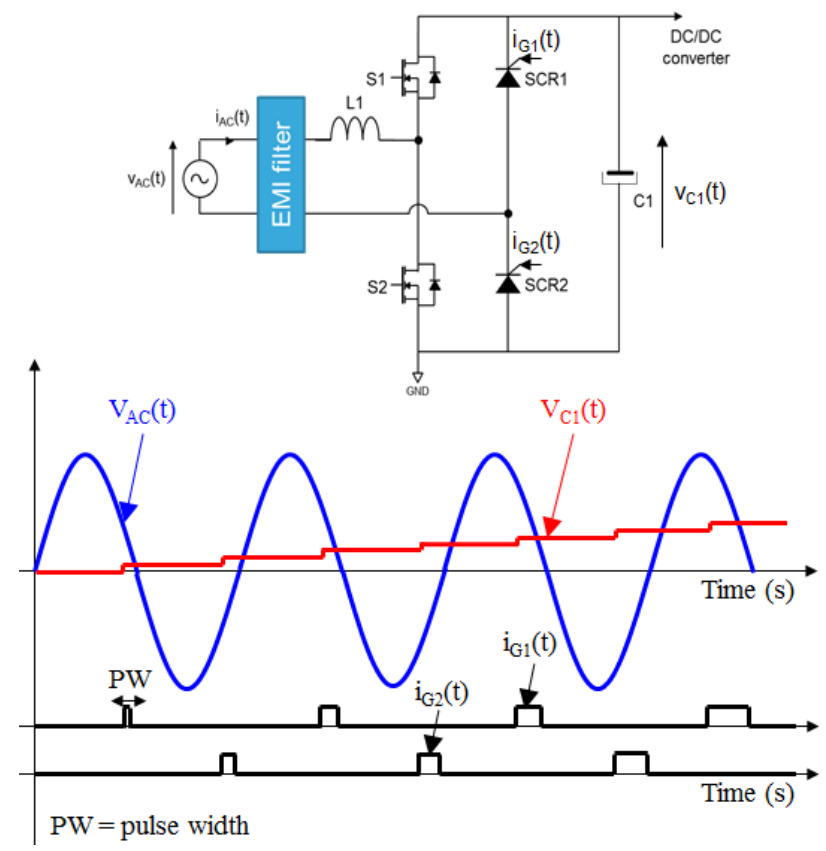

Fig. 2. Control strategy of the SCRs based on a constant triggering delay.

C. Reymond et al. have recently demonstrated that this control method of the SCRs is not totally satisfactory [9]. As can be seen in Figure 3, the charge of the DC-link capacitor (e.g., $1 \mathrm{mF}$ capacitance) lasts approximately $850 \mathrm{~ms}$. This value may be too important, particularly for automotive applications.
ICL based on a constant triggering delay $(50 \mu \mathrm{s})$ used to control the SCRs Test conditions: $230 \mathrm{~V}$ RMS, $50 \mathrm{~Hz}$ AC-line

DC-link capacitance $=1 \mathrm{mF}$

Charge duration $=850 \mathrm{~ms}$

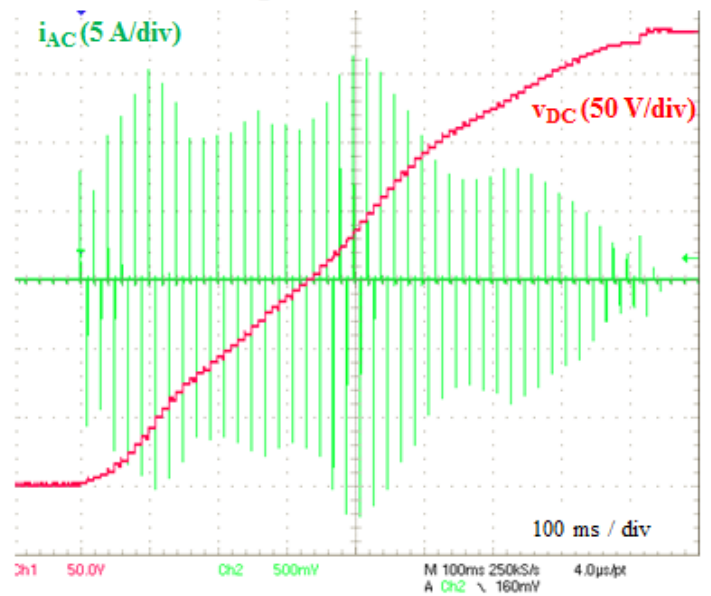

Fig. 3. Experimental test results of the SCRs' control strategy based on a constant triggering delay $(50 \mu \mathrm{s})$.

\section{Soft Start Optimization}

\section{A. SCR Control with a Variable Triggering Delay}

Another control strategy of the SCRs is based on an adjustable triggering delay $(\Delta t)$. The method consists in calculating the phase shift of each power device to reach a constant value of the peak current flowing through the AC-line. The main advantage is to accelerate the charge of the output capacitor (DC-link capacitor) during the AC-DC power supply startup.

Regarding this control strategy, the two SCRs are alternatively controlled according to the AC-line polarity by adjusting their turn-on delay. As can be seen in Figure 3, a modeling of the AC-DC converter is necessary to calculate (mathematical calculations) each triggering delay. Then, the power devices are controlled by a MCU thanks to an embedded look up table.

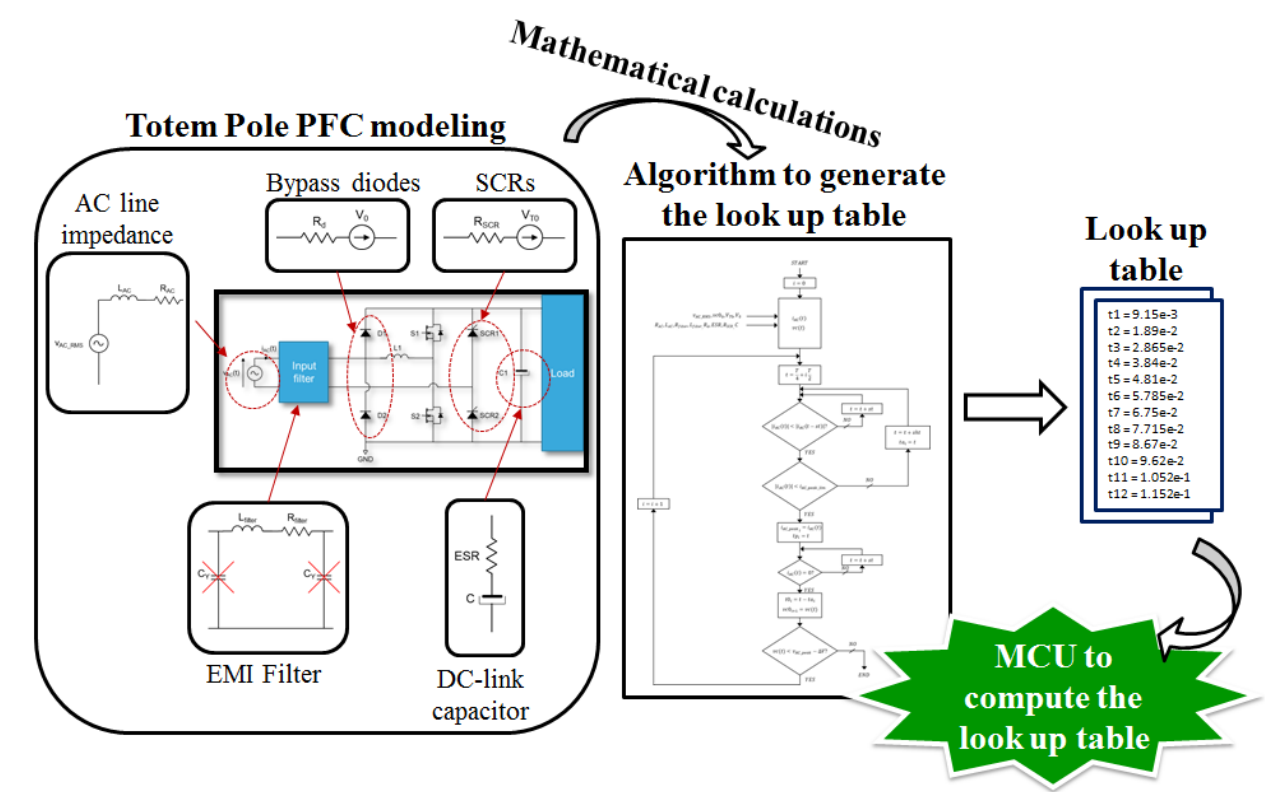

Fig. 4. Methodology used to control SCRs with an adjustable triggering delay. 


\section{B. Experimental Test Results}

An evaluation board was designed to prove the robustness of the totem pole PFC that embeds the inrush current limitation solution described above.

Figure 5 gives the comparison between mathematical simulation results (Mathcad engineering software tool) and experimental test results. In particular, the amplitude of inrush currents was limited by adjusting the triggering delay of each SCR. The AC-DC converter was plugged in
$230 \mathrm{~V}$ RMS, $50 \mathrm{~Hz}$ AC-mains. $1 \mathrm{mF}$ output capacitance (DC-link) was used.

Simulation and experimental test results (see Figure 5) show that the charge of the DC-link capacitor is accelerated using this control approach of the SCRs. In particular, the charge duration is about $120 \mathrm{~ms}$. This value is much lower than in the previous solution i.e., control of the SCRs using a constant triggering delay (using the same test conditions, the charge duration was about $850 \mathrm{~ms}$ ).
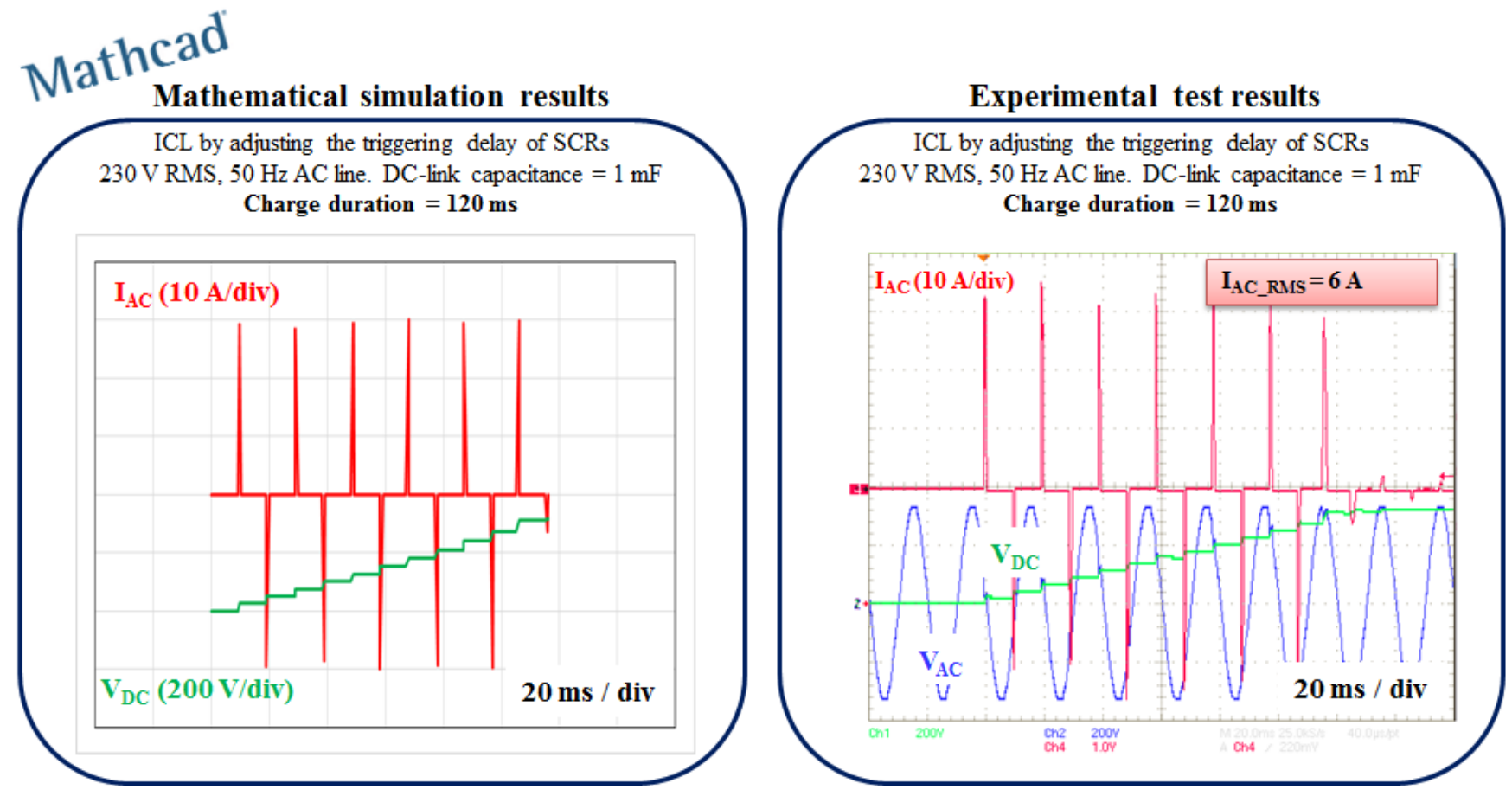

Fig. 5. Inrush current limiter by adjusting the SCRs' triggering delay. Comparison between mathematical simulation results (Mathcad engineering software tool) and experimental test results.

\section{Conclusions}

In this paper, an efficient and low-cost active inrush current limiter based on the smart control of SCRs is proposed. These SCRs are used in AC-DC power supplies dedicated to EV charging systems.

In particular, the control strategy consists in adjusting the triggering delay of SCRs both to limit the peak inrush currents and accelerate the charge of the DC-link capacitor of the AC-DC converter.

A modeling of the AC-DC converter was performed using mathematical calculations (the Mathcad engineering tool was used). The control strategy of each SCR was taken into consideration. The experimental test results demonstrate the robustness of the modeling.

The smart control of SCRs to limit inrush currents may be used in any type of AC-DC power supply. There are many applications: EV battery chargers, servers or lighting applications.

\section{References}

[1] N. C. dos Santos, C. E. Francischetti and R. Gomes da Silva, "Analysis of Electric Vehicles: a Brazilian Reality and its Consequences for the Environment", in Renewable Energy and Power Quality Journal, Vol. 1, No 12, 2014, pp. 195-200.

[2] Chandra Mukherjee and A. Gupta, "A Review of Charge Scheduling of Electric Vehicles in Smart Grid”, in IEEE Systems Journal, Vol. 9, No 4, 2015, pp. 1541-1553.

[3] M. F. M. Sabri, K. A. Danapalasingam and M. F. Rahmat, "A review on hybrid electric vehicles architecture and energy management strategies", in Renewable and Sustainable Energy Reviews, Vol. 53, 2016, pp. 1433-1442.

[4] Jamshid Aghaei, Ali Esmaeel Nezhad, Abdorreza Rabiee and Ehsan Rahimi, "Contribution of Plug-in Hybrid Electric Vehicles in power system uncertainty management", in Renewable and Sustainable Energy Reviews, Vol. 59, 2016, pp. 450458. 
[5] S. S. Williamson, A. K. Rathore and F. Musavi, "Industrial Electronics for Electric Transportation: Current State-of-the-Art and Future Challenges", in IEEE Transactions on Industrial Electronics, Vol. 62, No 5, 2015, pp. 3021-3032.

[6] B. Whitaker, A. Barkley, Z. Cole, B. Passmore, D. Martin, T. R. McNutt, A. B. Lostetter, J. Seung Lee and K. Shiozaki, "A High-Density, High-Efficiency, Isolated On-Board Vehicle Battery Charger Utilizing Silicon Carbide Power Devices", in IEEE Transactions on Power Electronics, Vol. 29, No 5, 2014, pp. 26062617.

[7] L. Xue, Z. Shen, D. Boroyevich, P. Mattavelli and D. Diaz, "Dual Active Bridge-Based Battery Charger for Plug-in Hybrid Electric Vehicle With Charging Current Containing Low Frequency Ripple", in IEEE Transactions on Power Electronics, Vol. 30, No 12, 2015, pp. 7299-7307.

[8] T. Ghanbari, E. Farjah, and A. Zandnia, "Development of a High-Performance Bridge-Type Fault Current Limiter", in IET Generation, Transmission \& Distribution, Vol. 8, No 3, 2014, pp. 486-494.

[9] C. Reymond, S. Jacques, G. Benabdelaziz and J.C. Lebunetel, "An Active Inrush Current Limiter Based on SCR Phase Shift Control for EV Charging Systems", in Journal of Energy and Power Engineering, Vol. 10, 2016, pp. 247-257.

[10] S. Abdel-Rahman, "Resonant LLC Converter: Operation and Design", Application Note AN 2012-09 V1.0, Infineon Technologies North America (IFNA) Corp, September 2012. 